\title{
Research Status of Traditional Chinese Medicine Constitution Theory in Insomnia
}

\author{
Chun-Hua ZHANG, Chun-Guang YU*, Pei-Yao LI, Ling WANG, \\ Hui-Xin DING, Wen-Wen ZHAO \\ Nursing College, Beijing University of Chinese Medicine, Beijing 100029, China
}

\begin{abstract}
With the development of modern bio-psycho-social medical model and traditional Chinese medicine (TCM) constitution theory, we gradually realize that the exploration of the constitution characteristics of insomnia is of great significance for the reveal of the origin of insomnia and the innovation of its treatment and nursing methods. This paper focused on the analysis of the investigation of TCM constitution classification of insomnia and the application of TCM constitution theory in the treatmentand nursing of insomnia. Moreover, the research prospect of insomnia in TCM constitution was discussed. The purpose is to provide theoretical basis for the treatment and nursing of patients with insomnia based on analysis of constitution differentiationin the future, and to better serve the clinic.
\end{abstract}

Keywords: Insomnia; TCM constitution; Treatment based on constitution differentiation/Constitution differentiation and treatment; Nursing based on constitution differentiation

\section{Introduction}

With the acceleration of the pace of social life and the increasing pressure caused by social competition and other reasons, the number of insomniacs has shown a rapid growth trend in recent years. In the United States, the number of outpatient visits due to insomnia symptoms increased by $13 \%$ from 1999 to $2010^{[1]}$. According to the survey released by the Chinese Sleep Research Society, the incidence of insomnia among adults in China is $38.2 \%$, which is significantly higher than that in developed countries $^{[2]}$. The adverse consequences of insomnia are serious threat to people's life and health, which may aggravate certain physical diseases or induce the onset of the original diseases. At the same time, these diseases

\footnotetext{
Corresponding Author:

Chun-Guang YU, Nursing College, Beijing University of Chinese Medicine, Beijing 100029, China E-mail: bjchunguang@163.com

Received:April 19 2019, Revised:April 20 2019,Accepted:April 25 2019, Online: May 152019
}

can aggravate the degree of insomnia, causing a vicious circle $^{[3]}$. In recent years, with the change of medical model and the development of TCM constitution theory, the connection between insomnia and TCM constitution has gradually attracted people's attention. People gradually realize that the exploration of the constitution characteristics of insomnia is of great significance for the reveal of the origin of insomnia and the innovation of its treatment and nursing methods. TCM constitution refers to the comprehensive and relatively stable intrinsic traits of morphological structure, physiological function and psychological state formed on the basis of congenital endowment and acquired environment in the course of human life. TCM constitution is also the human personality characteristics formed in the course of human growth and development that are compatible 
with the natural and social environment ${ }^{[4]}$. At present, the nine-point method of prestigious chinese physician Qi Wang $^{[5]}$ is widely used in the classification of constitution types. Based on this classification method, this paper will elaborate the research status of insomnia from the perspective of TCM constitution theory, in order to provide theoretical basis for clinical medical staff.

\section{Investigation on TCM Constitution of Insomnia}

\section{TCM constitution classification of Insomnia}

Constitution is inherited from birth and nurtured from acquired, and the formation and development of constitution are influenced by many factors. At present, there are relatively many clinical studies on the constitution classification of patients with insomnia, but the results are not identical. According to the literature, although the results are not identical, some of the constitution types tend to be consistent. Compared with the normal population, the insomniacs tend to have qistagnation constitution, qi-deficiency constitution, yindeficiency constitution, yang-deficiency constitution and phlegm-dampness constitution. For example, Pei et al. ${ }^{[6]}$ through a survey of 308 patients with insomnia, showed that the 3 most single constitutions were qi-deficiency type $(13.0 \%)$, yin-deficiency type (11.4\%) and phlegmdampness type (10.4\%); Zhao et al.$^{[7]}$ evaluated the 500 residents' health record registration forms in the Affiliated Hospital of Shanxi College of TCM, and concluded that insomnia was closely related to qi-deficiency constitution, qi-stagnation constitution, yin-deficiency constitution and damp-heat constitution. In addition, the constitution types also differs between genders. Studies ${ }^{[8-9]}$ have shown that qi-stagnation constitution is often more commonly occurring in women, and damp-heat constitution is more commonly occurring in men. The reason is that women are more likely to be have over-thinking, emotional sensitivity, more susceptible to external negative environmental influences, resulting in anxiety and depression. And depression is positively correlated with qi-stagnation constitution ${ }^{[10]}$, which can lead to insomnia. Men are more concerned with their work and social entertainments, often smoking and drinking, eating too much fried fatty food, causing dysfunction of the spleen and stomach, and producing endogenous damp heat and so no, thus forming damp-heat constitution.

Different age groups have different tasks, different living and working habits, thus the TCM constitution types of insomnia may be different. Through reviewing the literature we found, even in the same age group, there were differences in the constitution classification of college students, but in general, it was more common in qi-deficiency constitution and yin-deficiency constitution. Chen et al. ${ }^{[11]}$ investigated the incidence of chronic insomnia and the TCM constitution types of 110 students in Southern Medical University. The results showed that the main type of insomnia in Southern Medical University was qi-deficiency constitution. And Ling et al. ${ }^{[12]}$ investigated the insomniastatus of 1000 college students in Gansu University of TCM, and found that insomnia was closely related to yin-deficiency constitution, qi-deficiency constitution and yang-deficiency constitution. Cheng et $a l .^{[13]}$ through a survey of 1080 college students in 2 universities in Nanyang City, Henan Province, showed that yin-deficiency constitution, damp-heat constitution and qi-stagnation constitution were more commonly seen in the 386 students with insomnia. Although all of them are college students, the TCM constitution types of insomnia are not exactly the same. Considering the differences may be related to regional differences, differences in research sample size, academic pressure in different schools and so on, but qi-deficiency constitution and yin-deficiency constitution are more common. The most qi-deficiency constitution may be related to the college students' lack of exercise. There is more yin-deficiency constitution in college students, which may be related to the general lack of exercise, often staying up late, irregular diet, and preference for fried barbecue food, etc. In addition, compared with the constitution types of college students, the constitution of the modern elderly with insomnia is mainly deficiency. Xia et al. ${ }^{[14]}$ investigated the elderly in the Yangpu District of Shanghai, and found that deficient constitutions were more frequent than others in the elderly with insomnia, and yang-deficiency and qi-deficiency occurred mostly in unbalanced and simple constitutions. Peng et al. ${ }^{[15]}$ investigated the TCM constitution of 102 
elderly insomniacs in the First Affiliated Hospital of Guangxi University of TCM, and the results showed thatthe constitution of elderly patients with insomnia was mainly deficiency, including yang-deficiency constitution, yin-deficiency constitution, qi-deficiency constitution. It can be considered thatin old age, kidney essence is gradually exhausted, kidney yang is weak, and the generation of yangqi is lacking in source, so deficiency is the main performance. However, the results of Zuo et $a l .^{[16]}$ are different from these results. According to the survey of 168 elderly insomniacs in Dongzhimen Hospital, qi-stagnation constitution is the most common constitution, followed by phlegm-dampness constitution, yin-deficiency constitution and blood-stasis constitution. It can be considered that the most qi-stagnation constitution may be related to the lack of care from family for the elderly in the empty nest and are prone to anxiety and depression.

In addition, the constitution classification of insomnia in different regions may be different, which may be related to the geographical environment, lifestyle and human factors of different regions. Zhao et al. ${ }^{[17]}$ through the investigation of 900 patients with insomnia in Fangshan District of Beijing, they found that phlegm-dampness constitution (25.29\%), qi-stagnation constitution $(23.82 \%)$ and qi-deficiency constitution (15.74\%) were the most common constitution types. The reason for this may be that the climate in Beijing is cold, the yangqi is damaged, and the transportation and metaplasia are hindered. Because cold could make wet turbidity stagnation into phlegm turbidity, over time gradually formed phlegmdampness constitution; coupled with the fast pace of life in Beijing, the high pressure of work, consider these are the reasons for most qi-stagnation constitution. Li et $a l .^{[18]}$ surveyed 1200 insomniacs in Guangdong Province showed that qi-deficiency constitution (38.9\%) was the most common, followed by yang-deficiency constitution (32.6\%), damp-heat constitution (30.9\%) and qi-stagnation constitution $(30.1 \%)$. They believe that insomnia is highly prevalent in the urban senior intellectual youth, for they are in a high-intensity, high-stress environment for a long time, fatigue leads to qi consumption, visceral dysfunction, and formed qi deficiency, coupled with relatively hot in the south, it is easy to form qi deficiency because of the loss of qi followed sweat.

\section{Study on the difference of indexes among different constitutions of insomnia}

The study on the correlation between TCM constitution of insomnia and objective laboratory indicators can provide a basis for the objectification of clinical syndrome differentiation, and provide reference for clinical treatment and nursing. However, at present, there are relatively few studies on this aspect, and further studies on the correlation between different constitutions of insomnia and objective indicators need to be carried out in the future.

In order to study the relativity between electrical energy values of different meridians and the constitution types, She et al. ${ }^{[19]}$ investigated 169 patients with insomnia, and used the TCM meridian detector to detect the locations of Yuan-primary points in 12 meridians of the patients with different constitutions. It was found that the mean values of physical agility, metabolism, heart-kidney, physique changed to different degrees when the patients were with different constitutions. TCM attaches great importance to the relationship between human body constitution and disease. The constitution factors affect individual treatment and nursing response to a certain extent. The research on the correlation between electrical energy values of different meridians and constitution types can provide objective basis for the treatment and nursing based on clinical syndrome differentiationfor insomnia. Liang ${ }^{[20]}$ studied by using flow cytometry to detect lymphocyte subsets, NK cell killing function, NK cell subsets and granzyme, perforin, Th1, Th2, Th17 cell subsets; using enzyme-linked immuno sorbent assay (Elisa) to detect the IFN $\gamma$ and INF $\alpha$ levels secreted by NK cells in order to analyze the immune disorder status of chronic insomnia patients with yang-deficiency constitution from the perspective of NK cells and T cells. The results showed that there was a correlation between the degree of yang deficiency and the degree of the above indicators' changes, which provides a theoretical basis for microcosmic syndrome differentiation of chronic insomnia with yang-deficiency constitution, and provides 
a reference for treatment and nursing of chronic insomnia.

\section{Application of TCM Constitution Theory in the Treatment and Nursing of Insomnia}

At present, the application of TCM constitution theory in treatment and nursing of insomnia, most researchesare to verify the effectiveness of some intervention methods for patients with certain constitution types of insomnia, intervention methods which are commonly used in insomnia patients with qi-stagnation constitution and yindeficiency constitution.

\section{Treatment and nursing of insomnia patients with qi-stagnationconstitution}

Clinically, the treatment and nursing methods for insomnia patients with qi-stagnation constitution are relatively rich, including TCM treatment, acupuncture, auricular point sticking, music therapy, Chinese medicine foot bath and so on. And generally a variety of methods in combination were used, such as TCM treatment with music therapy, needling Si-guan point combined with auricular point sticking, Chinese medicine foot bath combined with auricular point sticking and other methods, with good clinical resultsachieved. For example, Zhang et al. ${ }^{[21]}$ used the method of body differentiation and regulation to treat the 48 patients with qi-stagnation constitution in the comprehensive group with the combination of Jue Tune music therapy and Chaihu Jia Longgu Muli Tang. After 4 weeks, the patient's qi stagnation status and the quality of sleep were significantly improved. Xie et al. ${ }^{[22]}$ studied the application of needling $\mathrm{Si}$-guan point and auricular point sticking on the basis of Chaihu Longmu Hehuan Tang, and relied on "Chaihu Longmu Hehuan decoction" as the main prescription for relieving the depressed liver and calming liver and suppressing liver-yang. Siguan point, namely point Hegu (li 4), point Taichong (lr 3), the two points pertain to yin and yang in nature respectively. Hegu is mainly regulating qi, responsible for rising, and Taichong is mainly regulating the blood, responsible for falling, so the combination of two points can realize the function ofregulating qi and blood. At the same time, auricular point sticking is applied to adjust the yin and yang of the organs, so as to achieve the purpose of treating and preventing diseases. In addition to needling Si-guan point, auricular point sticking is often combined with Chinese medicine foot bath. For example, $\mathrm{Hu}$ et $a l .{ }^{[23]}$ reported that 75 cases of insomnia patients with qi-stagnation constitution were treated with Chinese medicine foot bath and auricular point sticking based on constitution identification. After 4 nights, the totaleffective rate is as high as $93.3 \%$, and the effect is remarkable. In addition, the expert experience has also achieved good development. He et al. ${ }^{[24]}$ have summarized the clinical experience of Professor Jia in the treatment of refractory insomnia. Professor Jia believes that the cause of refractory insomnia is mostly caused by qi stagnation. Based on this, he proposes a three-step treatment program for heart and brain treatment, mental conditioning and assisted therapy: Chinese medicine treatment (Ningshen Anzang decoction) combined with mental care plus convenient and effective TCM nursing techniques, such as auricular point sticking, acupoint massage, cupping therapyand so on, together to achieve the role of prolonging the patient's sleep time and improving the quality of sleep.

\section{Treatment and nursing of insomnia patients with yin-deficiency constitution}

Yin-deficiency constitution is one of the common types of constitution in patients with insomnia. In this type of constitution, the body fluid is in a relatively lacking state for a long time. At night, yin deficiency causes the body to fail to converge Yangqi normally, the yang is too excited, causing insomnia. And in turn, longterm insomnia will also aggravate the loss of body fluid, forming a vicious circle ${ }^{[25]}$. Clinical interventions for insomnia patients with yin-deficiency constitution often involve TCM treatment, auricular point sticking, acupuncture and other methods. For example, $\mathrm{Xu}$ et al. ${ }^{[26]}$ reported that, through constitution identification, 44 cases of insomnia patients with yin-deficiency constitution were treated with oral administration of Suanzaoren decoction, $150 \mathrm{~mL} / \mathrm{d}, 2$ times/d, in the morning and evening, the total effective rate reached $88.89 \%$ after 14 days, and compared with Western medicine, there were no significant adverse reactions, the quality of life of patients 
significantly improved. In addition, Li et al. ${ }^{[25]}$ used needle warming therapy to intervene in insomnia patients with yin-deficiency constitution. Through constitution identification, 35 cases of insomnia patients with yindeficiency constitution were treated with conventional acupuncture therapy combined with needle warming therapy. After 3 courses of treatment, the symptoms of insomnia were significantly improved. At the same time, the research is also a response to the debate about "whether hot diseases can be treated with moxibustion?". The researchers believe that for yin deficiency generating inner heat, moxibustion can be used by warming and invigorating yangqi, yin and yang interdependence, and mutual promotion of growth between yin and yang, to achieve the role of replenishing yin fluid, so insomnia patients with yin-deficiency constitution can be treated with needle warming therapy.

\section{Treatment and nursing of insomnia patients with other constitution types}

In addition to the more common qi-stagnation constitution and yin-deficiency constitution in the constitution types of insomnia patients, some studies have also discussed the clinical interventions of constitution types such as yang-deficiency, qi-deficiency and blood-stasis. Deng et $a{ }^{[27]}$ used TCM foot bath fumigation and washing to treat insomnia patients with yang-deficiency constitution, stimulated the acupoints of the feet through the foot bath, meanwhile, the blood flow was increased by the warm effect of water, and the medicine was brought into the body through the skin and the meridians, so as to achieve the role of warming the movement of yangqi, regulating blood and vessel, making balance between yin and yang, and sleeping well at night. Jin et al. ${ }^{[28]}$ explored the effect of "Yiqi Anshen Ointment" in the treatment of qi-deficiency constitution insomnia based on the "fiveviscera Correlation" theory. Insomnia locates in the heart, but it is closely related to liver, lung, spleen and kidney. Insomnia can be caused by any one of the five viscera dysfunction. The researchers have developed the "Yiqi Anshen Ointment" through the combination of herbs with different nature and taste, meridian distribution. It has the effect of reconciling the relationship between five viscera. It has a good effect on insomnia patients with qi-deficiency combination. Wang et al. ${ }^{[29]}$ used scraping therapy based on the theory of midnight-noon ebb-flow to treat 43 cases of perimenopausal insomnia women with phlegm-dampness constitution, and the scraping therapy was conducted every Monday, Wednesday and Friday. The results showed that this intervention method could improve the patient's sleep quality, but also improve the patient's perimenopausal hot flashes, sweating, backache and other symptoms.

\section{Outlook and Development}

Constitution is a person's inherent characteristics, with relative stability. Using the perspective of TCM Constitution theory, trying to find the high-risk constitution type of insomnia, syndrome differentiation and treatment based on patients' constitution, and adjusting the constitution biasembody the individualized diagnosis and treatment thinking of TCM. It can reach the purpose of "preventing a disease before its onset" and "preventing a disease from changing and exacerbating". Moreover, the TCM constitution theory is of great significance for disease prevention, health preserving and individualized diagnosis and treatment and nursing of different individual constitutions ${ }^{[30]}$. Xu et al ${ }^{[30]}$ took 40 insomnia patients with cardiovascular disease as the experimental group to observe the effects of comprehensive lifestyle interventionsunder the guidance of preventive treatment on them. The interventions included exercise guidance, dietary guidance and TCM constitution conditioning guidance. The results showed that the insomnia and other symptoms of patients in this group were significantly improved, which played the advantage of TCM "preventing a disease before its onset, and preventing a disease from changing and exacerbating". Therefore, improving the comprehensive lifestyle of insomnia patients with different biased constitutions through interventions under the guidance of "preventive treatment of disease" can be the future research direction.

Through literature review, we find out that although the research on TCM constitution of insomnia has been made some progress, the application of TCM constitution 
theory in the study of insomnia patients is still not mature enough and perfect. At present, the intervention methods for insomnia are mainly according to treatment and nursing based on syndrome differentiation. For example, both Guo et al. ${ }^{[32]}$ and Wang et al. ${ }^{[33]}$ have treated and nursed insomnia through syndrome differentiation. There are relatively few documents on the application of differentiation and treatment with constitution, and fewer documents on the application of differentiation and nursing with constitution. Therefore, in the treatment and nursing of insomnia, differentiation and treatment with constitution, differentiation and nursing with constitution as a new idea, it can provide reference for our future research direction.

In addition, genomicsand metabolomics have been gradually applied to the study of TCM constitution in recent years. And some scholars have carried out some exploratory researches using genomics and metabolomics $^{[34]}$. So in the future we can also try to explore the treatment of insomnia by changing the expression of insomnia genes. At the same time, foreign studies have shown that behavioral insomnia is a very common problem throughout childhood, while domestic research is more inclined to the study of young, middle and old people, and less research on children's insomnia, so future exploratory studies on constitution of insomnia children can also be carried out ${ }^{[35]}$.

\section{Summary}

Insomnia is an alternating disorder of normal rhythm of sleep and wakefulness, a physical and mental disease that causes a decline of sleep quality. It is a common clinical manifestation of various physical, mental and behavioral diseases. Long-term insomnia can cause a decline of social function and quality of life ${ }^{[36]}$. With the change of lifestyle and the increase of life pressure, the number of insomniacs is increasing. In the process of diagnosis and treatment of insomnia, the unique advantages oftreatment based on syndrome differentiation, treatment based on constitutiondifferentiation, nursing based on constitutiondifferentiation, and the concept of wholism in TCM have gradually emerged. From the article we can see that everyone's attention to the TCM constitution of insomnia is getting higher and higher, and there are relatively many studies on the classification of TCM constitution in insomnia, but the research results are not exactly the same. The reason may be related to the difference in sample size of the study. Besides, it may be related to the differences in age, gender and region of insomnia patients included in the study. At the same time, in the study of the correlation between TCM constitution of insomnia and objective laboratory indicators, we find out that there are relatively few studies in this area, which can be used as a research direction in the future. On the one hand, understanding the differences in the expression of various constitutional laboratory indicators can provide an objective basis for the classification of constitution of insomnia; on the other hand, understanding the degree of correlation between different constitutions and laboratory indicators can provide an important reference for TCM treatment and nursing based on syndrome differentiation of insomnia. In addition, although TCM constitution theory has played an important role in the treatment and nursing for insomnia, and has made some progress, more studies are to verify the effectiveness of some intervention methods for insomnia patients of certain constitution types. There is a lot of work to be done. Finally, we hope that in the future, we can fully apply the ideas of treatment and nursing based on constitution differentiation in the field of insomnia, and try to explore the relationship between insomnia genomics and constitution, and hope to find out the high-risk constitution types of insomnia by using the viewpoint of TCM constitution theory and to give early interventions, so as to achieve the goal of "preventing a disease before its onset" and "preventing a disease from changing and exacerbating".

\section{Declaration}

The authors of this article declare they have no conflict of interest.

\section{References}

1 Ford ES, Wheaton AG, Cunningham TJ, et al. Trends in Outpatient Visits for Insomnia, Sleep Apnea, 
and Prescriptions for Sleep Medications among US Adults: Findings from the National Ambulatory Medical Care Survey 1999-2010. Sleep, 2014, 37(8): 1283-1293.

2 Zhao ZX, Zhang ZH. More attention should be paid to sleep. Chin J Neurol, 2011, 44(8): 513-515.

3 Zhao ZX. Clinical sleep disorder diagnosis and treatment manual. Shanghai: The Second Military Medical University Press, 2006: 225-255.

4 China Association of Chinese Medicine. Classification and Determination of Constitution in TCM. Beijing: China Press of Traditional Chinese Medicine, 2009.

5 Wang Q.TCM Constitution 2008. Beijing: People's Medical Publishing House, 2009: 104.

6 Pei QH, Li X, Xu J, et al. Study on the correlation between TCM constitution and sleep quality of 308 patients with primary insomnia. J Beijing Univ Tradit Chin Med, 2018, 41(10): 859-862.

7 Zhao LN, Jia YJ. Study on the relationship between insomnia and TCM constitution.Chin J Integr Med on Cardio/Cereb Dis, 2015, 13(12): 1448-1450.

8 Ma XH, Sun HR. TCM body constitution Analysis of 95 cases of sub-health insomnia patients. J Shaanxi Coll Tradit Chin Med, 2017, 40(1): 36-38.

9 Huang QY, Luo HL, Li XQ, et al. Investigation on 172 cases of insomnia patients with TCM constitution. J New Chin Med, 2015, 47(8): 30-32.

10 Liu MF, Jiang Y, Wang XM, et al. The Role of Rumination and Stressful Life Events in the Relationship between the Qi Stagnation Constitution and Depression in Women: A Moderated Mediation Model. Evid Based Complement Alternat Med, 2017, (6): 1-8.

11 Chen Y, Shen SQ, Hu GF. Study on the Relationship of Traditional Chinese Medical Constitution and Chronic Insomnia. West China Med J, 2016, 31(5): 901-904.

12 Ling J, Song YJ, Liu SR, et al. Correlation between insomnia and Traditional Chinese Medical Constitution classification of college students. J Gansu Coll Tradit Chin Med, 2015, 32(4): 91-94.

13 Cheng L, Li Z, Zhang W, et al. Correlation between insomnia and types of traditional Chinese medicine constitution in college students. Chin J School Health,
2018, 39(10): 1499-1501.

14 Xia C, Zhu QB, Huang F, et al. Traditional Chinese medicine constitution types in 127 elderly patients with insomnia: an investigation in communities of Yangpu District, Shanghai. J Integr Med, 2012, 10(8): 866-873.

15 Peng X, Han JB, Ning YY, et al. TCM constitution analysis and nursing countermeasures of elderly insomnia patients.Chin General Pract Nurs, 2013, 11(5): 1157-1158.

16 Zuo JC. Correlation analysis between constitution and TCM syndromes in elderly patients with insomnia. Beijing: Beijing University of Chinese Medicine, 2013.

17 Zhao TB, Hu YX, Wen YP, et al. Investigation of Chinese Medicine Physical Epidemiology in Insomnia Population in Fangshan District. World J Sleep Med, 2018, 5(9): 1005-1009.

18 Li EM, Yin JP, Ning XX, et al. Investigation of Constitution and Meridian Energy Values for Subhealth State with Insomnia Population in Guangdong Area. Guid J Tradit Chin Med Pharm, 2018, 24(13): 69-70.

19 She SY, Yang MN, Chen X, et al. The Study on the Relativity of Insomnia Constitution Types and Auxiliary Diagnosis of Meridian Detector. Western J Tradit Chin Med, 2017, 30(10): 122-124.

20 Liang HY.Characteristics of NK cells and T cells in patients with chronic insomniadue to Yang Deficiency. Guangzhou: Guangzhou University of Chinese Medicine, 2017.

21 Zhang Y, Shen YJ, Hu w, et al. Clinical Observation on 48 Cases of Insomnia of Qi Stagnation Type Treated by Jue Tone Music Combined with Chaihu Jia Longgu Muli Tang. J Tradit Chin Med, 2017, 58(18): 1577-1580.

22 Xie XH, Wen LX, Zhang YZ, et al. Clinical Observation on Intervention of Kai Siguan and Auricular Point Sticking in the treatment of qi stagnation Constitution Insomnia. Chin Foreign Med Res, 2018, 16(33): 31-33.

$23 \mathrm{Hu}$ YM, Miao J, Zhang XT. Therapeutic effect of foot bath combined with Auricular Point Sticking on 140 patients with insomnia of qi stagnation Constitution. 
World Chin Med, 2016, 11(B03): 1282-1283.

24 Hei XY, Jia MH, Jia XS, et al. Clinical Experience of Jia Menghui Treating Intractable Insomnia. AsiaPacific Tradit Med, 2017, 13(15): 74-76.

25 Li WX. Clinical Study on needle warming therapy for Treatment of Yin DeficiencyConstitution Type Insomnia. Guangzhou: Guangzhou University of Chinese Medicine, 2014.

$26 \mathrm{Xu}$ JS, Liu JH, Yu ZY. Clinical observation on the therapeutic effect of treating Yin-deficient people with insomnia with Suan Zao Ren decoction: 44 cases. Chin J Basic Med Tradit Chin Med, 2013, 19(12): 1491-1493.

27 Deng YH, Zhou NH, Zhou JY, et al. Randomized controlled study of traditional Chinese medicine foot bath fumigation and washing for Insomnia of Yang deficiency Constitution. Zhejiang J Tradit Chin Med, 2017, 52(1): 21-22.

28 Jin Y, Chen RF, Chang SQ, et al. Therapeutic effect of Yiqi Anshen ointment in treating Qi deficiency insomnia. Hainan Med J, 2018, 29(22): 3126-3128.

29 Wang ZY. Clinical observation ofmidnight-noon and ebb-flow doctrine and Scraping in the treatment of Perimenopausal Insomnia of Phlegm dampness Constitution. Chengdu: Chengdu University of TCM, 2015 .
30 Zhou FY, Chen X. Status of Traditional Chinese Medicine Constitutional Theory in Diabetes Mellitus. China J Chin Med, 2015, 30(3): 343-345.

31 Xu HY, Zhang ZY. The Effect of Lifestyle Intervention on High Risk Population of Cardiovascular Combined with Insomnia Disease. World J Sleep Med, 2017, 4(5): 290-294.

32 Guo YF, Lu H, Gu WZ, et al. Clinical Study on Lianghe Decoction in Treating Insomnia with Liver Qi Stagnation and Yin Deficiency Syndrome. World Chin Med, 2016, 11(12): 2608-2610.

33 Wang LL, Liao M, Ye XN, et al. Curative effect of TCM-based syndrome differentiation combined with diet nursing and point massage on insomnia. Mod Clin Nurs, 2014, 13(7): 24-27.

34 Wang J, Li YS, Li LR, et al. Genomics study on nine types of TCM constitution. Chin J Tradit Chin Med Pharm, 2014, 29(12): 3871-3873.

35 Tikotzky L, Sadeh A. The role of cognitive-behavioral therapy in behavioral childhood insomnia. Sleep Med, 2010, 11(7): 686-691.

$36 \mathrm{Xu}$ FP. Observation on the Therapeutic Effect and Mechanism of Shuxin Anshen Ointment in Treating Insomnia of Yang Deficiency Constitution. Guangzhou: Guangzhou University of Chinese Medicine, 2014. 